\title{
Relative Performance of Different Colour Laden Sticky Traps on the Attraction of Sucking Pests in Pomegranate
}

\author{
K. Elango ${ }^{1 *}$, S. Sridharan ${ }^{1}$, P.A. Saravanan ${ }^{1}$ and S. Balakrishnan ${ }^{2}$ \\ ${ }^{1}$ Department of Agricultural Entomology, ${ }^{2}$ Department of Spices and Plantation Crops, Tamil \\ Nadu Agricultural University, Coimbatore, Tamil Nadu, India \\ *Corresponding author
}

\section{A B S T R A C T}

Different colour sticky traps are commonly used for monitoring the population of many sucking pests such as thrips, whiteflies, leaf miner adults and aphids. They have also been used to monitor the natural enemies associated with the sucking pests. Presently, the

\begin{tabular}{|l|}
\hline Ke y w or d s \\
Pomegranate, Sticky \\
traps, Sucking pests, \\
Yellow, Aphis punicae \\
\hline Article Info \\
\hline Accepted: \\
24 September 2017 \\
Available Online: \\
10 November 2017 \\
\hline \hline
\end{tabular}
monitoring the incidence of sucking pests in pomegranate using sticky traps is wanting. Hence, the study was conducted in pomegranate planting at orchard of Tamil Nadu Agricultural University, Coimbatore during 2016, using different colour laden sticky traps like yellow, pale yellow, green, orange and red. The traps were placed at five different locations and every week, the location of trap is changed and the attraction of aphids, thrips, whiteflies and coccinellids to traps is recorded. Green was the most attracted colour for aphids (208.4 aphid adults /per trap/per week) followed by yellow (202.6 aphid adults /per trap/per week) and pale yellow. While yellow was most attractive for thrips (177.4 thrips/per trap/per week) and white fly (22.1 whiteflies /per trap/per week) followed by pale yellow (122.1 thrips/per trap/per week) \& (13.8 whiteflies/per trap/per week) and green (110.9 thrips/per trap/per week) \& (13.1 whiteflies/per trap/per week) respectively. Most insect predators like lady bird beetles, minute pirate bugs, parasitic wasps and honey bees also exhibited preferences to particular trap colour characteristics, whereas green lacewings and spiders showed no such preference.

\section{Introduction}

Pomegranate (Punica granatum L.) is an important fruit crop of arid and semiarid regions of world. It is believed to have originated from Iran. India is one of the leading producers of pomegranate in world (Pal et al., 2014). In Tamil Nadu, the concept of high density planting and ultra-high density planting is being introduced and followed in pomegranate and mango. Pomegranate is cultivated under high density planting in important districts of Tamil Nadu viz., Coimbatore, Erode, Tiruppur and Karur. The cultivation of crop under high density demands the study on the level occurrence of various sucking pests. Cultivation of high yielding cultivars of pomegranate with intensive care and management in the recent past under irrigated conditions with early stage exploitation of plants has led to certain severe pest problems. Among them, infestation by sucking pests like aphids, thrips, whiteflies, mealy bugs, scale insects and mites results in reduction of pomegranate fruit yield and quality. The major constraint in 
increasing export potential is the quality of fruit in terms of size, colour, free from blemishes and pesticide residue levels. Pest control decisions are often based on trap catches as a cultural control measure. Insects are differentially attracted to coloured surfaces, particularly yellow as a general insect attractant; a feature exploited among entomologists for collection of Coleoptera, Hemiptera, Hymenoptera and Thysanoptera (Riley and Schuster, 1994; Kersting and Baspinar 1995).

\section{Materials and Methods}

\section{Evaluation of different colour laden sticky traps on the attraction of sucking pests of pomegranate}

The study was taken during January 2016 to March 2016 because the peak activity of pomegranate aphids was observed during the second fortnight of December. The highest infestation of thrips was noticed in the second fortnight of March. Population density of whiteflies was maximum during the second fortnight of February and the first fortnight of March reported by Kotikal et al., (2011). The sticky traps of area $(0.3 \mathrm{~m} \times 0.2 \mathrm{~m})$ in five different colours viz., yellow, pale yellow, green, orange and red were tested for monitoring sucking pests population on pomegranate. This field experiment was conducted in TNAU orchard, Coimbatore to fix ideal colour for monitoring the sucking pests population (Fig. 1).

Normal sticky trap available with private companies costs is Rs 50/trap which will be effective for a maximum period of seven days during the period the trap will be adhered with insect, scales and dust making it in effective. Hence, a cost effective sticky trap using the laminated colour sheet coated with grease was developed. It can be re used by removing the grease and the trapped insects.
The laminated colour sheet $(0.3 \mathrm{~m} \times 0.2 \mathrm{~m})$ costs Rs 15/- only making it cost effective and efficient. Commercial grade grease was smeared as an adhesive on the outer surface of the sticky trap while was fixed to the ground using wooden stake equidistantly in the pomegranate field.

The traps were placed at five different locations and every week, the location of trap was changed and the attraction of adult aphids, thrips and whiteflies to traps was recorded. Observation was continued upto 10 weeks. The data was pooled and analyzed statistically.

\section{Statistical analysis}

The data gathered in the field experiment was analyzed by using AGRES 3.01 and AGDATA software. The population of insect pests was subjected to square root transformations. Duncan multiple range test (DMRT) was applied for comparing treatment means at 5 per cent level of significance (Duncan, 1951).

\section{Results and Discussion}

\section{Monitoring of thrips (Scirtothrips dorsalis) using colour sticky traps}

The data on attraction of $S$. dorsalis to colour sticky traps at weekly intervals is given in Table 1. The data presented in the Table 1 showed that yellow coloured sticky trap significantly attracted more number of thrips with a mean population of 177.4 thrips per trap per week. The other best treatment in attracting the thrips population was pale yellow (122.1 thrips per trap per week) followed by green colour (110 thrips per trap per week). The orange and red coloured sticky traps were least effective in attracting the thrips with a mean population of 92.70 and 60.00 numbers respectively (Fig. 2). 
Monitoring of aphid (Aphis punicae) using colour sticky traps

The data presented in the table 2 showed the effectiveness of colour sticky traps in attracting A. punicae population at weekly intervals. Among the different colour sticky traps tested, green colour attracted significantly higher number of aphids with a mean population of 208.4 aphids per trap per week followed by yellow stick trap with the mean population 202.6 aphids (Fig. 3).

Both green and yellow colour sticky traps showed significant attraction of aphids over other colour traps over a period of 10 weeks observation. Whereas, other sticky traps like pale yellow, orange and red colours recorded a mean population of $154.4,150.4$ and 103.4 aphids per trap per week respectively.

\section{Monitoring of whitefly (Siphoninus phillyreae) using colour sticky traps}

Yellow coloured sticky trap significantly attracted more number of whiteflies with a mean population of 22.1 whiteflies per trap per week. Next best treatment in attracting the whitefly population was pale yellow (13.8 whiteflies per trap per week) followed by green colour (13.1 whiteflies per trap per week). The orange and red coloured sticky traps were least effective in attracting the whiteflies with a mean population of 10.6 and 6.9 respectively. The data presented in the Table 3 (Fig. 4).

Fig.1 Evaluation of different colour laden sticky traps for sucking pests of pomegranate

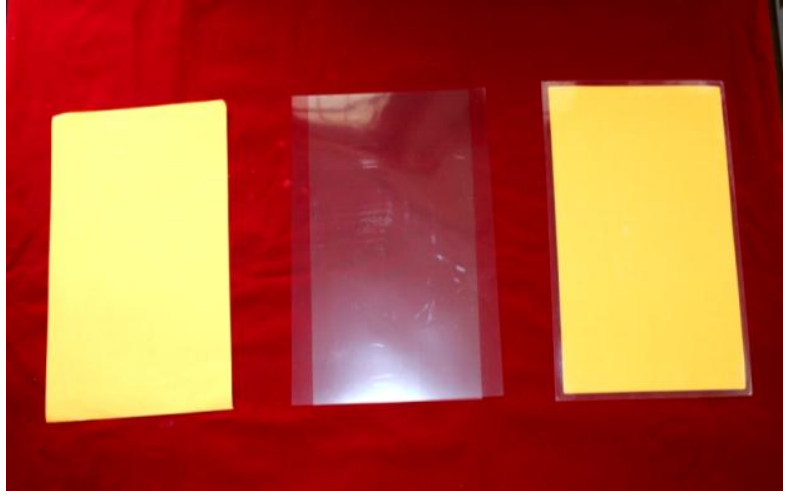

a. Laden colour sticky trap

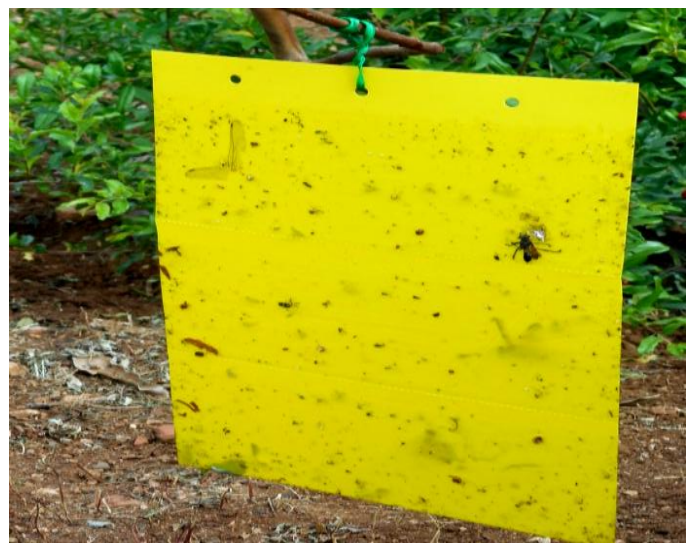

c. Yellow sticky trap attraction

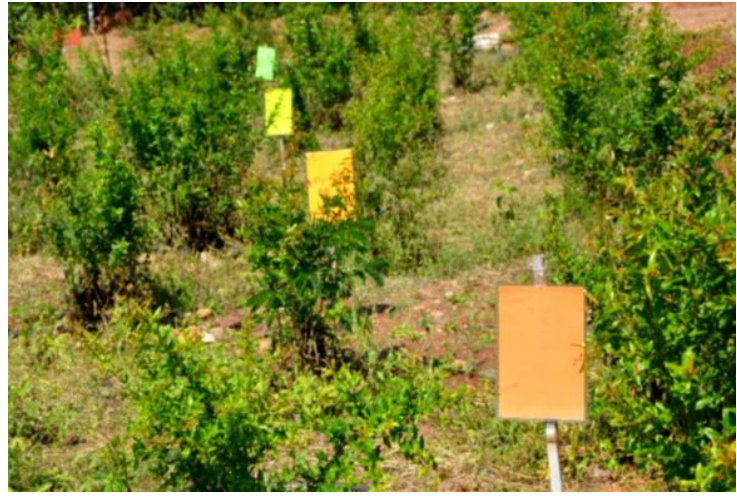

b. Sticky traps in field

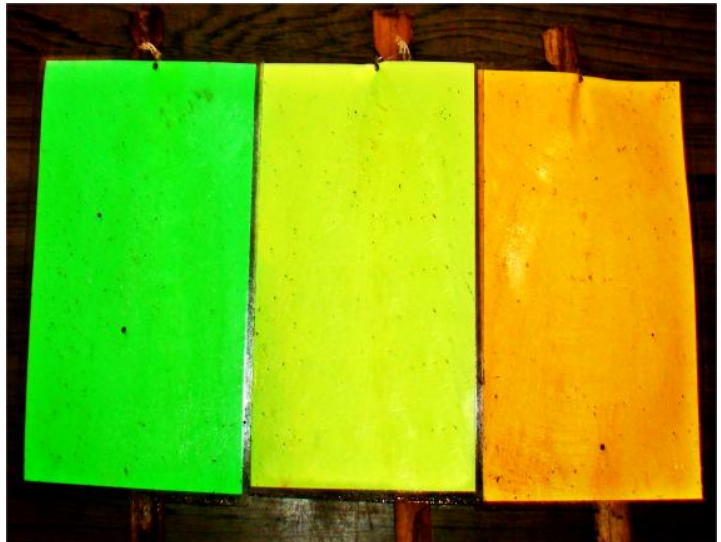

d. Laden colour sticky trap attraction 
Fig.2 Trapping efficiency of different colour sticky traps on thrips

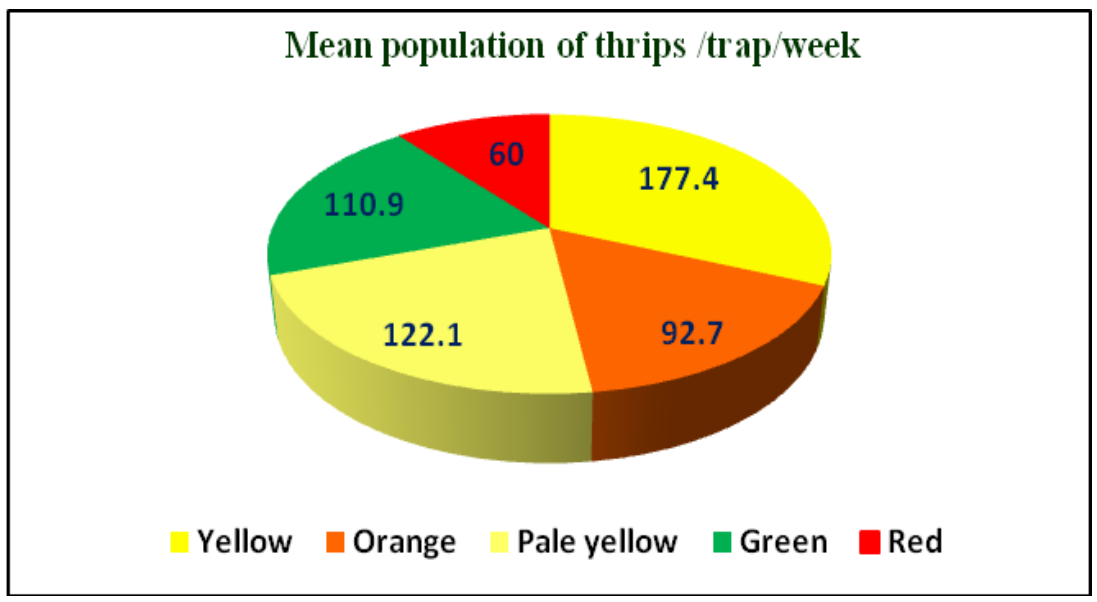

Fig.3 Trapping efficiency of different colour sticky traps on aphids

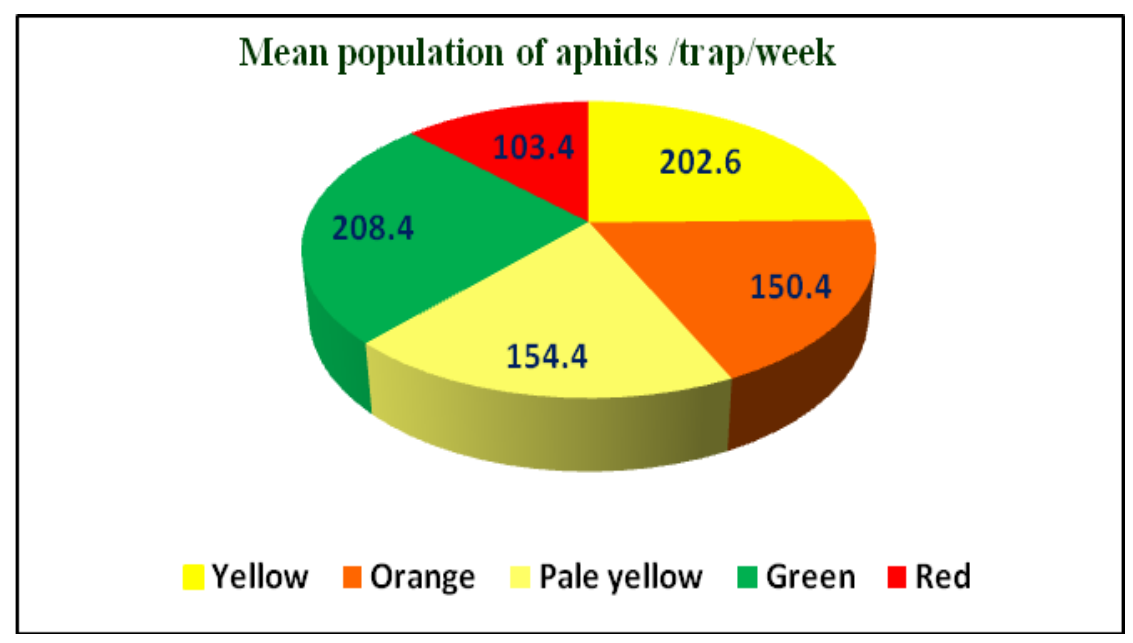

Fig.4 Trapping efficiency of different colour sticky traps on whitefly

Mean population of whitefly/trap/week

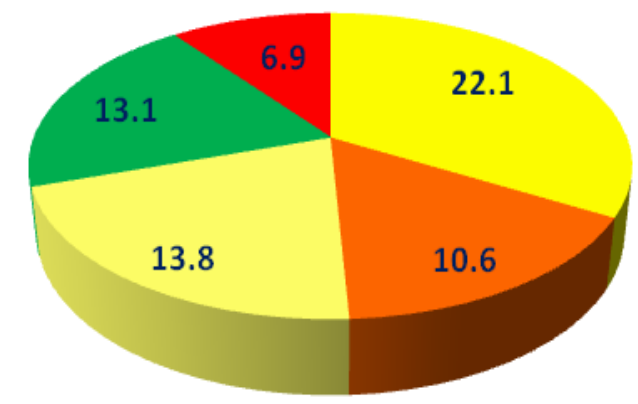

Yellow Orange Pale yellow Green $\square$ Red 
Table.1 Trapping efficiency of different colour sticky traps on thrips (Scirtothrips dorsalis)

\begin{tabular}{|c|c|c|c|c|c|c|c|c|c|c|c|}
\hline \multirow{3}{*}{$\begin{array}{c}\text { Colour of sticky } \\
\text { traps }\end{array}$} & \multicolumn{10}{|c|}{ No. of thrips /trap /week* } & \multirow{3}{*}{ Mean } \\
\hline & \multicolumn{3}{|c|}{ January } & \multicolumn{4}{|c|}{ February } & \multicolumn{3}{|c|}{ March } & \\
\hline & $2^{\text {nd }}$ week & $3^{\text {rd }}$ week & $4^{\text {th }}$ week & $1^{\text {st }}$ week & $2^{\text {nd }}$ week & $3^{\text {rd }}$ week & $4^{\text {th }}$ week & $1^{\text {st }}$ week & $2^{\text {nd }}$ week & $3^{\text {rd }}$ week & \\
\hline $\begin{array}{c}\text { Yellow } \\
(565-590 \mathrm{~nm})\end{array}$ & $\begin{array}{c}243 \\
(15.58)^{\mathrm{a}}\end{array}$ & $\begin{array}{c}186 \\
(13.68)^{\mathrm{a}}\end{array}$ & $\begin{array}{c}149 \\
(12.20)^{\mathrm{a}}\end{array}$ & $\begin{array}{c}116 \\
(10.77)^{\mathrm{a}}\end{array}$ & $\begin{array}{c}161 \\
(12.68)^{\mathrm{a}}\end{array}$ & $\begin{array}{c}186 \\
(13.63)^{\mathrm{a}}\end{array}$ & $\begin{array}{c}202 \\
(14.31)^{\mathrm{a}}\end{array}$ & $\begin{array}{c}192 \\
(13.88)^{\mathrm{a}}\end{array}$ & $\begin{array}{c}173 \\
(13.15)^{\mathrm{a}}\end{array}$ & $\begin{array}{c}166 \\
(12.88)^{\mathrm{a}}\end{array}$ & 177.4 \\
\hline $\begin{array}{c}\text { Orange } \\
(590-625 \mathrm{~nm})\end{array}$ & $\begin{array}{c}92 \\
(9.59)^{\mathrm{d}}\end{array}$ & $\begin{array}{c}84 \\
(9.16)^{\mathrm{d}}\end{array}$ & $\begin{array}{c}68 \\
(8.24)^{\mathrm{d}}\end{array}$ & $\begin{array}{c}74 \\
(8.60)^{\mathrm{d}}\end{array}$ & $\begin{array}{c}102 \\
(10.09)^{\mathrm{d}}\end{array}$ & $\begin{array}{c}78 \\
(8.83)^{\mathrm{d}}\end{array}$ & $\begin{array}{c}92 \\
(9.89)^{\mathrm{d}}\end{array}$ & $\begin{array}{c}112 \\
(10.58)^{c}\end{array}$ & $\begin{array}{c}131 \\
(11.44)^{b}\end{array}$ & $\begin{array}{c}94 \\
(9.69)^{\mathrm{d}}\end{array}$ & 92.7 \\
\hline $\begin{array}{l}\text { Pale yellow } \\
(570-585 \mathrm{~nm})\end{array}$ & $\begin{array}{c}102 \\
(10.09)^{\mathrm{c}}\end{array}$ & $\begin{array}{c}86 \\
(9.27)^{c}\end{array}$ & $\begin{array}{c}103 \\
(10.14)^{\mathrm{b}}\end{array}$ & $\begin{array}{c}93 \\
(9.64)^{\mathrm{c}}\end{array}$ & $\begin{array}{c}132 \\
(11.48)^{b}\end{array}$ & $\begin{array}{c}148 \\
(12.16)^{b}\end{array}$ & $\begin{array}{c}152 \\
(12.32)^{b}\end{array}$ & $\begin{array}{c}136 \\
(11.66)^{b}\end{array}$ & $\begin{array}{c}112 \\
(10.58)^{c}\end{array}$ & $\begin{array}{c}157 \\
(12.52)^{\mathrm{b}}\end{array}$ & 122.1 \\
\hline $\begin{array}{c}\text { Green } \\
(520-565 \mathrm{~nm})\end{array}$ & $\begin{array}{c}131 \\
(11.44)^{\mathrm{b}}\end{array}$ & $\begin{array}{c}116 \\
(10.77)^{\mathrm{b}}\end{array}$ & $\begin{array}{c}88 \\
(9.38)^{\mathrm{c}}\end{array}$ & $\begin{array}{c}103 \\
(10.14)^{b}\end{array}$ & $\begin{array}{c}122 \\
(11.04)^{\mathrm{c}}\end{array}$ & $\begin{array}{c}92 \\
(9.59)^{\mathrm{c}}\end{array}$ & $\begin{array}{c}118 \\
(10.86)^{c}\end{array}$ & $\begin{array}{c}97 \\
(9.84)^{\mathrm{d}}\end{array}$ & $\begin{array}{c}108 \\
(10.39)^{\mathrm{c}}\end{array}$ & $\begin{array}{c}134 \\
(11.57)^{\mathrm{c}}\end{array}$ & 110.9 \\
\hline $\begin{array}{c}\text { Red } \\
(625-740 \mathrm{~nm})\end{array}$ & $\begin{array}{c}48 \\
(6.92)^{\mathrm{e}}\end{array}$ & $\begin{array}{c}56 \\
(7.48)^{\mathrm{e}}\end{array}$ & $\begin{array}{c}62 \\
(7.87)^{\mathrm{e}}\end{array}$ & $\begin{array}{c}72 \\
(8.48)^{\mathrm{d}}\end{array}$ & $\begin{array}{c}42 \\
(6.48)^{\mathrm{e}}\end{array}$ & $\begin{array}{c}83 \\
(9.11)^{\mathrm{c}}\end{array}$ & $\begin{array}{c}55 \\
(7.41)^{\mathrm{e}}\end{array}$ & $\begin{array}{c}49 \\
(7.00)^{\mathrm{e}}\end{array}$ & $\begin{array}{c}61 \\
(7.81)^{\mathrm{d}}\end{array}$ & $\begin{array}{c}72 \\
(8.48)^{\mathrm{e}}\end{array}$ & 60 \\
\hline SE d & 0.0840 & 0.0413 & 0.0507 & 0.0636 & 0.0450 & 0.0390 & 0.1002 & 0.0862 & 0.9485 & 0.0845 & $\begin{array}{ll}---- \\
--1\end{array}$ \\
\hline $\mathrm{CD}$ & 0.1830 & 0.0900 & 0.1106 & 0.1386 & 0.0981 & 0.0849 & 0.2184 & 0.1879 & 2.0665 & 0.1841 & ---- \\
\hline
\end{tabular}

*Mean of four replications; figures in parentheses are square root transformed values; in a column, means followed by a common letter(s) are not significantly different by DMRT $(\mathrm{P}=0.05)$

Table.2 Trapping efficiency of different colour sticky traps on aphids (Aphis punicae)

\begin{tabular}{|c|c|c|c|c|c|c|c|c|c|c|c|}
\hline \multirow{3}{*}{$\begin{array}{c}\text { Colour of sticky } \\
\text { traps }\end{array}$} & \multicolumn{10}{|c|}{ No. of aphids /trap/week* } & \multirow{3}{*}{ Mean } \\
\hline & \multicolumn{3}{|c|}{ January } & \multicolumn{4}{|c|}{ February } & \multicolumn{3}{|c|}{ March } & \\
\hline & $2^{\text {nd }}$ week & $3^{\text {rd }}$ week & $4^{\text {th }}$ week & $\mathbf{1}^{\mathrm{sr}}$ week & $2^{\text {nd }}$ week & $3^{\text {rd }}$ week & $4^{\text {th }}$ week & $1^{\text {st }}$ week & $2^{\text {nd }}$ week & $3^{\text {rd }}$ week & \\
\hline $\begin{array}{c}\text { Yellow } \\
(565-590 \mathrm{~nm})\end{array}$ & $\begin{array}{c}288 \\
(16.97)^{\mathrm{a}}\end{array}$ & $\begin{array}{c}228 \\
(15.09)^{b}\end{array}$ & $\begin{array}{c}211 \\
(14.52)^{\mathrm{a}}\end{array}$ & $\begin{array}{c}284 \\
(16.85)^{\mathrm{a}}\end{array}$ & $\begin{array}{c}220 \\
(14.83)^{\mathrm{a}}\end{array}$ & $\begin{array}{c}116 \\
(10.77)^{\mathrm{c}}\end{array}$ & $\begin{array}{c}173 \\
(13.15)^{\mathrm{b}}\end{array}$ & $\begin{array}{c}134 \\
(11.57)^{\mathrm{b}}\end{array}$ & $\begin{array}{c}206 \\
(14.35)^{b}\end{array}$ & $\begin{array}{c}166 \\
(12.88)^{\mathrm{b}}\end{array}$ & 202.6 \\
\hline $\begin{array}{c}\text { Orange } \\
(590-625 \mathrm{~nm})\end{array}$ & $\begin{array}{c}163 \\
(12.76)^{\mathrm{d}}\end{array}$ & $\begin{array}{c}154 \\
(12.40)^{c}\end{array}$ & $\begin{array}{c}161 \\
(12.69)^{b}\end{array}$ & $\begin{array}{c}150 \\
(12.24)^{\mathrm{c}}\end{array}$ & $\begin{array}{c}163 \\
(12.76)^{c}\end{array}$ & $\begin{array}{c}141 \\
(11.87)^{\mathrm{b}}\end{array}$ & $\begin{array}{c}127 \\
(11.26)^{\mathrm{c}}\end{array}$ & $\begin{array}{c}138 \\
(11.74)^{\mathrm{b}}\end{array}$ & $\begin{array}{c}186 \\
(13.63)^{\mathrm{c}}\end{array}$ & $\begin{array}{c}121 \\
(11.00)^{\mathrm{d}}\end{array}$ & 150.4 \\
\hline $\begin{array}{l}\text { Pale yellow } \\
(570-585 \mathrm{~nm})\end{array}$ & $\begin{array}{l}181 \\
(13.45) \mathrm{c}\end{array}$ & $\begin{array}{c}172 \\
(13.11)^{\mathrm{c}}\end{array}$ & $\begin{array}{c}153 \\
(12.36)^{\mathrm{c}}\end{array}$ & $\begin{array}{c}186 \\
(13.63)^{b}\end{array}$ & $\begin{array}{c}151 \\
(12.28)^{\mathrm{d}}\end{array}$ & $\begin{array}{c}149 \\
(12.20)^{\mathrm{b}}\end{array}$ & $\begin{array}{c}118 \\
(10.86)^{\mathrm{d}}\end{array}$ & $\begin{array}{c}140 \\
(11.83)^{\mathrm{b}}\end{array}$ & $\begin{array}{c}161 \\
(12.68)^{d}\end{array}$ & $\begin{array}{c}133 \\
(11.53)^{\mathrm{c}}\end{array}$ & 154.4 \\
\hline $\begin{array}{c}\text { Green } \\
(520-565 \mathrm{~nm})\end{array}$ & $\begin{array}{c}256 \\
(16.00)^{\mathrm{b}}\end{array}$ & $\begin{array}{c}243 \\
(15.58)^{\mathrm{a}} \\
\end{array}$ & $\begin{array}{c}227 \\
(15.06)^{\mathrm{a}} \\
\end{array}$ & $\begin{array}{c}198 \\
(14.07)^{b}\end{array}$ & $\begin{array}{c}204 \\
(14.28)^{b} \\
\end{array}$ & $\begin{array}{c}182 \\
(13.49)^{\mathrm{a}} \\
\end{array}$ & $\begin{array}{c}191 \\
(13.82)^{\mathrm{a}}\end{array}$ & $\begin{array}{c}177 \\
(13.30)^{\mathrm{a}} \\
\end{array}$ & $\begin{array}{c}225 \\
(15.00)^{\mathrm{a}}\end{array}$ & $\begin{array}{c}181 \\
(13.45)^{\mathrm{a}} \\
\end{array}$ & 208.4 \\
\hline $\begin{array}{c}\text { Red } \\
(625-740 \mathrm{~nm})\end{array}$ & $\begin{array}{c}115 \\
(10.72)^{\mathrm{e}}\end{array}$ & $\begin{array}{c}121 \\
(11.00)^{\mathrm{e}}\end{array}$ & $\begin{array}{c}88 \\
(9.38)^{\mathrm{d}}\end{array}$ & $\begin{array}{c}92 \\
(9.59)^{\mathrm{d}}\end{array}$ & $\begin{array}{c}110 \\
(10.48)^{\mathrm{e}}\end{array}$ & $\begin{array}{c}83 \\
(9.11)^{\mathrm{d}}\end{array}$ & $\begin{array}{c}91 \\
(9.53)^{\mathrm{e}}\end{array}$ & $\begin{array}{c}106 \\
(10.29)^{\mathrm{c}}\end{array}$ & $\begin{array}{c}132 \\
(11.48)^{\mathrm{e}}\end{array}$ & $\begin{array}{c}96 \\
(9.79)^{\mathrm{e}}\end{array}$ & 103.4 \\
\hline SE d & 0.0588 & 0.1401 & 0.3299 & 0.4649 & 0.0992 & 0.1832 & 0.0843 & 0.3151 & 0.0717 & 0.0331 & ----- \\
\hline $\mathrm{CD}$ & 0.1281 & 0.3054 & 0.7188 & 1.0130 & 0.2160 & 0.3992 & 0.1836 & 0.6866 & 0.1563 & 0.0722 & --.-- \\
\hline
\end{tabular}

*Mean of four replications; figures in parentheses are square root transformed values; in a column, means followed by a common letter(s) are not significantly different by DMRT $(\mathrm{P}=0.05)$ 
Table.3 Trapping efficiency of different colour sticky traps on whitefly (Siphoninus phillyreae)

\begin{tabular}{|c|c|c|c|c|c|c|c|c|c|c|c|}
\hline \multirow{3}{*}{$\begin{array}{c}\text { Colour of } \\
\text { sticky traps }\end{array}$} & \multicolumn{10}{|c|}{ No. of whitefly/trap/week } & \multirow{3}{*}{ Mean } \\
\hline & \multicolumn{3}{|c|}{ January } & \multicolumn{4}{|c|}{ February } & \multicolumn{3}{|c|}{ March } & \\
\hline & $2^{\text {nd }}$ week & $3^{\text {rd }}$ week & $4^{\text {th }}$ week & $1^{\text {st }}$ week & $2^{\text {nd }}$ week & $3^{\text {rd }}$ week & $4^{\text {th }}$ week & $1^{\text {st }}$ week & $2^{\text {nd }}$ week & $3^{\text {rd }}$ week & \\
\hline $\begin{array}{c}\text { Yellow } \\
(565-590 \mathrm{~nm})\end{array}$ & $\begin{array}{c}43 \\
(6.55)^{\mathrm{a}}\end{array}$ & $\begin{array}{c}31 \\
(5.56)^{\mathrm{a}}\end{array}$ & $\begin{array}{c}28 \\
(5.29)^{\mathrm{a}}\end{array}$ & $\begin{array}{c}22 \\
(4.69)^{\mathrm{a}}\end{array}$ & $\begin{array}{c}16 \\
(4.00)^{\mathrm{a}}\end{array}$ & $\begin{array}{c}10 \\
(3.16)^{\mathrm{a}}\end{array}$ & $\begin{array}{c}12 \\
(3.46)^{\mathrm{a}}\end{array}$ & $\begin{array}{c}21 \\
(4.58)^{\mathrm{a}}\end{array}$ & $\begin{array}{c}24 \\
(4.89)^{\mathrm{a}}\end{array}$ & $\begin{array}{c}14 \\
(3.74)^{\mathrm{a}}\end{array}$ & 22.1 \\
\hline $\begin{array}{c}\text { Orange } \\
(590-625 \mathrm{~nm})\end{array}$ & $\begin{array}{c}18 \\
(4.24)^{\mathrm{c}}\end{array}$ & $\begin{array}{c}11 \\
(3.31)^{d}\end{array}$ & $\begin{array}{c}13 \\
(3.60)^{\mathrm{c}}\end{array}$ & $\begin{array}{c}16 \\
(4.00)^{c}\end{array}$ & $\begin{array}{c}7 \\
(2.64)^{d}\end{array}$ & $\begin{array}{c}4 \\
(2.00)^{\mathrm{d}}\end{array}$ & $\begin{array}{c}6 \\
(2.44)^{\mathrm{e}}\end{array}$ & $\begin{array}{c}8 \\
(2.82)^{\mathrm{d}}\end{array}$ & $\begin{array}{c}11 \\
(3.31)^{d}\end{array}$ & $\begin{array}{c}12 \\
(3.46)^{b}\end{array}$ & 10.6 \\
\hline $\begin{array}{l}\text { Pale yellow } \\
(570-585 \mathrm{~nm})\end{array}$ & $\begin{array}{c}24 \\
(4.89)^{b}\end{array}$ & $\begin{array}{c}12 \\
(3.46)^{\mathrm{c}}\end{array}$ & $\begin{array}{c}20 \\
(4.47)^{\mathrm{b}}\end{array}$ & $\begin{array}{c}14 \\
(3.74)^{d}\end{array}$ & $\begin{array}{c}9 \\
(3.00)^{c}\end{array}$ & $\begin{array}{c}7 \\
(2.64)^{b}\end{array}$ & $\begin{array}{c}11 \\
(3.31)^{b}\end{array}$ & $\begin{array}{c}16 \\
(4.00)^{b}\end{array}$ & $\begin{array}{c}14 \\
(3.71)^{\mathrm{c}}\end{array}$ & $\begin{array}{c}11 \\
(3.31)^{b}\end{array}$ & 13.8 \\
\hline $\begin{array}{c}\text { Green } \\
(520-565 \mathrm{~nm})\end{array}$ & $\begin{array}{c}19 \\
(4.35)^{\mathrm{c}}\end{array}$ & $\begin{array}{c}16 \\
(4.00)^{\mathrm{b}}\end{array}$ & $\begin{array}{c}12 \\
(3.46)^{\mathrm{d}}\end{array}$ & $\begin{array}{c}18 \\
(4.24)^{b}\end{array}$ & $\begin{array}{c}14 \\
(3.74)^{b}\end{array}$ & $\begin{array}{c}6 \\
(2.44)^{c}\end{array}$ & $\begin{array}{c}9 \\
(3.00)^{c}\end{array}$ & $\begin{array}{c}11 \\
(3.31)^{\mathrm{c}}\end{array}$ & $\begin{array}{c}18 \\
(4.24)^{\mathrm{b}}\end{array}$ & $\begin{array}{c}8 \\
(2.82)^{c}\end{array}$ & 13.1 \\
\hline $\begin{array}{c}\text { Red } \\
(625-740 \mathrm{~nm})\end{array}$ & $\begin{array}{c}2 \\
(1.41)^{d}\end{array}$ & $\begin{array}{c}6 \\
(2.44)^{\mathrm{e}}\end{array}$ & $\begin{array}{c}14 \\
(3.74)^{\mathrm{c}}\end{array}$ & $\begin{array}{c}7 \\
(2.64)^{\mathrm{e}}\end{array}$ & $\begin{array}{c}5 \\
(2.23)^{\mathrm{e}}\end{array}$ & $\begin{array}{c}3 \\
(1.73)^{\mathrm{e}}\end{array}$ & $\begin{array}{c}8 \\
(2.82)^{d}\end{array}$ & $\begin{array}{c}11 \\
(3.31)^{c}\end{array}$ & $\begin{array}{c}9 \\
(3.00)^{\mathrm{e}}\end{array}$ & $\begin{array}{c}4 \\
(2.00)^{d}\end{array}$ & 6.9 \\
\hline SE d & 0.0749 & 0.0565 & 0.0461 & 0.0516 & 0.0686 & 0.0874 & 1.0396 & 0.0552 & 0.0515 & 0.0698 & ------ \\
\hline $\mathrm{CD}$ & 0.1632 & 0.1231 & 0.1005 & 0.1123 & 0.1495 & 0.1904 & 2.2651 & 0.1202 & 0.1122 & 0.1520 & ----- \\
\hline
\end{tabular}

*Mean of four replications; figures in parentheses are square root transformed values; in a column, means followed by a common letter(s) are not significantly different by DMRT $(\mathrm{P}=0.05)$ 
A simple and efficient monitoring tool for sucking pests is sticky trap. In the present investigation, the descending order of thrips attraction to colour sticky traps observed were yellow $>$ pale yellow $>$ green $>$ orange $>$ red. More numbers of thrips were attracted to yellow coloured sticky traps than other colours. The attraction of thrips to orange and red were least. The more attracting yellow coloured sticky traps shall be efficiently used in pomegranate to assess the activity of thrips, so that effective management practices may be followed to manage the thrips population. The present observations were in conformity with the earlier researchers who worked on sticky traps for monitoring of thrips. Aphid attraction to colour sticky traps were green $>$ yellow > pale yellow > orange > red. More number of aphids was attracted to green colour sticky traps than other colours. Yellow coloured sticky trap significantly attracted more number of whiteflies in the pomegranate eco system. Similarly, Premalatha and Rajangam (2011) reported that maximum number of whitefly Trialeurodes vaporariorum (Westwood) (adults) attracted towards yellow sticky trap in gerbera. Likewise Lu et al., (2012) reported that yellow sticky traps can be used as an effective method for the control of whiteflies, Bemisia tabaci in the greenhouse. These reports support the present finding.

Yellow coloured sticky traps attracted maximum number of thrips and whitefly with a mean population of 177.4 and 22.1 per trap per week. Green coloured sticky traps attracted the maximum number of aphid population of 208.4 per trap per week. So in pomegranate field different colour laden sticky traps are effective for monitoring the population of many sucking pests such as thrips, whiteflies adults and aphids. The laminated colour sheet is cost effective and efficient in monitoring of sucking pests and their natural enemies.

\section{Acknowledgement}

The authors are grateful to Dean, School of Post Graduate Study (SPGS), Director, Professor and Head, Department of Entomology and Central for Plant Protection Studies and Tamil Nadu Agricultural University for giving assistance during my research period.

\section{References}

Duncan, D.B. 1951. A significant test for differences between ranked treatment means in an analysis of variance. Virginia Journal of sciences, 2: 171 189.

Kersting, U. and Baspinar, H. 1995. Seasonal and diurnal flight activity of Circulifer haematoceps (Hom. Cicadellidae), an important leafhopper vector in the Mediterranean area and the Near East. Journal of Applied Entomology, 119: 533-537.

Kotikal, Y.K., N. Ananda and R.A. Balikai. 2011. Seasonal Incidence of Major Sucking Pests of Pomegranate and Their Relation with Weather Parameters in India. Proc. II ${ }^{\text {nd }}$ IS on Pomegranate and Minor, including Mediterranean fruits. Eds.M.K. Sheikh et al., Acta Hort., 589-596.

Lu, Y., Bei, Y., Zhang, J., and Ni, X. (2012). Are yellow sticky traps an effective method for control of sweetpotato whitefly, Bemisia tabaci, in the greenhouse or field?. Journal of Insect Science, 12(1).

Pal, R. K., Babu, K. D., Singh, N. V., Maity, A., and Gaikwad, N. (2014). Pomegranate Research in India-Status and future challenges. Progressive Horticulture, 46(2), 184-201.

Premalatha, K. and J. Rajangam. 2011. Efficacy of yellow sticky traps against greenhouse whitefly, Trialeurodes 
vaporariorum

(Aleyrodidae: Hemiptera) in Gerbera.

Journal of Biopesticides, 4 (2): 208-

210.
(Westwood)
Riley, D. G. and Schuster, D. J. 1994. Pepper weevil adult response to colored sticky traps in pepper fields. Southwestern Entomologist, 19: 93-107.

\section{How to cite this article:}

Elango, K., S. Sridharan, P.A. Saravanan and Balakrishnan, S. 2017. Relative Performance of Different Colour Laden Sticky Traps on the Attraction of Sucking Pests in Pomegranate. Int.J.Curr.Microbiol.App.Sci. 6(11): 2997-3004. doi: https://doi.org/10.20546/ijcmas.2017.611.350 